8th International Symposium on

Precision Engineering Measurements and Instrumentation

\title{
ISPEMI 2012
}

\section{Call for papers}
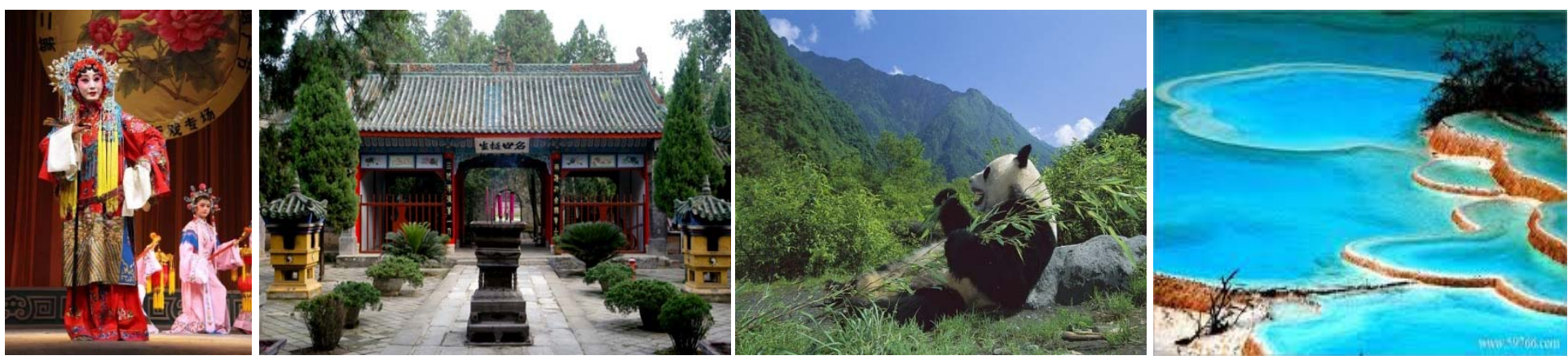

\section{8-11 August 2012 I Chengdu, China}

Sponsors

International Committee on Measurements and Instrumentation (ICMI)

National Natural Science Foundation of China (NSFC)

Chinese Society for Measurement (CSM)

China Instrument and Control Society (CIS)

Organizers

International Committee on Measurements and Instrumentation (ICMI) Instrumentation Committee of CSM Harbin Institute of Technology (HIT)

Cooperating Organizations

SPIE

Institute of Optics and Electronics, Chinese Academy of Sciences

Hefei University of Technology (HFUT)

Beijing Information Science and Technology University

Conference homepage

http://www.ispemi.org/
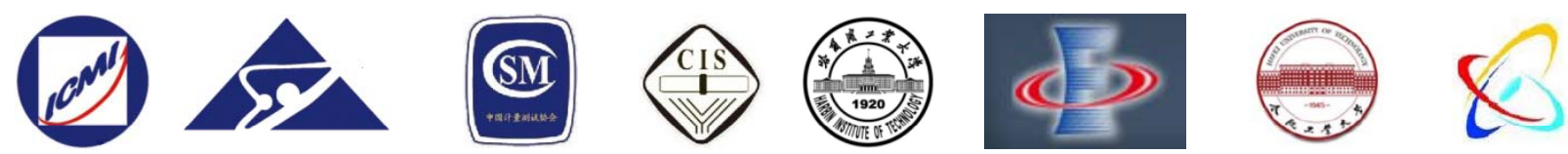


\title{
ISPEMI 2012
}

\section{8th International Symposium on}

\section{Precision Engineering Measurements and Instrumentation}

\begin{abstract}
International Symposium on Precision Engineering Measurements and Instrumentation (ISPEMI) is an international symposium held every other year in different cities of China with English as its working language since 1999. ISPEMI 2012 is the 8th ISPEMI. It is sponsored by International Committee on Measurements and Instrumentation (ICMI), National Natural Science Foundation of China (NSFC), Chinese Society for Measurement (CSM), and China Instrument and Control Society (CIS), and organized by Harbin Institute of Technology (HIT), together with Institute of Optics and Electronics, Chinese Academy of Sciences, SPIE, Hefei University of Technology, and Beijing Information Science and Technology University. The purpose of ISPEMI 2012 is to bring together young and senior researchers and engineers working in the field of precision engineering measurements and instrumentation both inside and outside China, and provide an international technical forum for them to present their researches and develop their knowledge of the recent advances on this particular aspect.
\end{abstract}

Oral and post sessions are planned for ISPEMI 2012 in the following subject areas

- Instrumentation Theory and Methodology

- Measurement for Precision and Ultra-Precision Machining

- $\quad$ Novel Instrument and Measurement System

- Modern Optics and Instruments for Precision Measurement

- $\quad$ Sensors, Converters, and Control System

- $\quad$ Optoelectronic System and Optical Instruments Design

- $\quad$ Laser Measurement Techniques and Instruments

- Instrument and Measurement System Calibration

- $\quad$ MEMS and Nanometer Measurement

- $\quad$ Accuracy Theory and Uncertainty Analysis

- Measurement for Advanced Optics Machining

It is also worth mentioning that all the papers contained in the SPIE Proceeding of previous meeting are indexed by El and other overseas indexing services. 


\section{8-11 August 2012 Chengdu, China}

ISPEMI 2012

8th International Symposium on

\section{Precision Engineering Measurements and Instrumentation}

\section{General requirements for papers}

In order to help with the publication of papers, ISPEMI 2012 has officially signed an agreement with SPIE to appoint SPIE as the publisher for publication of the proceedings of ISPEMI 2012.

It is clearly stated in the agreement signed that the proceedings shall be in English and the papers shall be six to ten pages long, single line spaced and single columned.

According to the agreement reached,

1) The organizing committee for ISPEMI 2012 shall select and referee all material submitted by authors and ensure that the technical quality of the final manuscripts is strong and that they conform to the SPIE guidelines for style and clarity of language;

2) SPIE shall perform quality assurance on the metadata and submitted manuscripts to ensure that they meet all the formatting standards and requirements;

3) Authors shall be responsible for correcting problems and submitting a revised manuscript via My SPIE in a timely manner. Any manuscript that is not formatted according to SPIE written specifications, is not written in clear English or does not reach SPIE on time may not be included in the proceedings.

\section{Important Note: PEER REVIEW}

All submissions will be peer reviewed by the full program committee. In order to facilitate the evaluation of your submission, please submit both a short $200-$ words abstract that will be normally with a full-length paper, and a 500-words structured extended abstract by the Abstract and Extended Abstract Due Date. The structured extended abstract can be up to three pages in length and should have figures, and sufficient data to permit review of the paper. To accommodate the peer review process, abstracts and extended abstracts will not be accepted after 15 March, 2012.

\section{Critical Dates:}

Abstract Due Date

15th March, 2012

Manuscript Due Date

1st July, 2012 $\star$ Please Note: Submissions imply the intent of at least one author to register, attend the symposium, present the paper as scheduled, and a full-length manuscript for publication in the conference proceedings.

Submit your abstract today!

\section{Venue: \\ - Chengdu}

Chengdu is the capital of Sichuan province, of Southwest China. Chengdu is also one of the most important economic centres, transportation and communication hubs in Western China. More than four thousand years ago, the prehistorical Bronze Age culture of Jinsha established itself in this region. The fertile Chengdu Plain, on which Chengdu is located, is also known as the country of heaven. Chengdu was recently named China's 4th-most livable city by China Daily.

\section{Further Information}

Please use the following information to contact us:

Post Add: P.O.Box 3017, Science Park, Harbin Institute of Technology, No. 2 Yikuang Street, NanGang District, Harbin 150080, China

Tel/Fax: +86-451-86402258

Website: http://www.ispemi.org/

E-mail: ispemi2012@hotmail.com 


\section{ISPEMI 2012}

\section{8th International Symposium on \\ Precision Engineering Measurements and Instrumentation}

\section{Honorary chairs}

Academician Guofan Jin, Tsinghua University (China)

Academician Tongbao Li, Tongji University (China)

Academician Zhonghua Zhang, NIM (China)

Academician Jie Gao, Sichuan University (China)

Academician Shenghua Ye, Tianjin University (China)

Academician Wenhan Jiang, Institute of Optics and Electronics (IOE), Chinese Academy of Sciences, (China)

Academician Xiangdi Lin, Institute of Optics and Electronics (IOE), Chinese Academy of Sciences, (China)

Academician Yuri V. Chugui, TDI SIE, Siberian Branch of the Russian Academy of Sciences (Russia)

Prof. Zhu Li, Huangzhong University of Science and Technology (China)

Prof. Guoxiong Zhang, Tianjin University (China)

Prof. Ahmed Abou-zeid, Physikalisch-Technische Bundesanstalt (Germany)

Prof. Peter Rolfe, University of Genova (Italy)

Dr. Konrad Herrmann, Physikalisch-Technische Bundesanstalt (Germany)

Prof. Chao-Kuang Chen, National Cheng Kung University (Taiwan, China)

\section{Chairman}

Prof. Jiubin Tan, Harbin Institute of Technology (HIT), China

\section{Co-chairs}

Prof. Tony Wilson, University of Oxford (U.K.)

Prof. Harald Bosse, PTB (Germany)

Prof. Min Gu, Swinburne University of Technology (Australia)

Prof. K. C. Fan, National Taiwan University (China)

Prof. Wei Gao, Tohoku University (Japan)

Prof. Seung-Woo Kim, KAIST (Korea)

\section{Chairman of Programme Committee}

Prof. Jiubin Tan, Harbin Institute of Technology (HIT), China

\section{Chairman of Organizing Committee}

Prof. Yudong Zhang, Institute of Optics and Electronics (IOE), Chinese Academy of Sciences (Chengdu, China) 